\title{
RECHERCHES TECHNIQUES SUR DES BRONZES DE GAULE ROMAINE, II*
}

\author{
par M. PICON, J. CONDAMIN, S. BOUCHER
}

A côté des bronzes de type gréco-romain, précédemment étudiés, on rencontre en Gaule romaine deux séries d'objets, les bronzes de type alexandrin et ceux de type gallo-romain, qui montrent certains caractères propres que nous définirons ici. Par commodité, nous adoptons la nomenclature qui présente le mérite de différencier nettement les objets gréco-romains, classiques par leur plastique et leur idéologie, et les objets gallo-romains, redevables à l'esthétique classique mais correspondant à des idées religieuses locales.

\section{BRonzes DE TYPE "ALEXANDRIN"}

Nous avons été amenés à en aborder l'étude à la suite de l'examen de deux d'entre eux, dont l'un semblait être la copie moderne de l'autre (analyses nos 45 et 87, cf. plus bas, n. 9 et p. 159). Le problème s'est révélé particulièrement intéressant, et nous avons étudié tous les objets répondant à cette dénomination qui se trouvent à Lyon dans les Musées de la Civilisation gallo-romaine de Fourvières et du Palais des Beaux-Arts, auxquels nous avons joint une statuette de Vienne (analyse $\mathrm{n}^{\circ}$ 80). Nous avons introduit sous leur ancien numéro les analyses qui avaient été effectuées précédemment.

Le terme "alexandrin" désigne une catégorie d'objets qui se différencient des objets grecs et romains par leur inspiration exotique ou caricaturale. Si l'on excepte un certain nombre de statues de grandes ou moyennes dimensions (bronzes de Mahdia ${ }^{1}$, pêcheur de Volubilis², etc.), ils sont représentés le plus souvent par des statuettes (nègres, grotes-

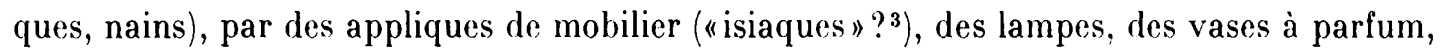
des décors d'ustensiles (manches de couteaux, poignées de lampes). Beaucoup de ces objets se rencontrent en Egypte ${ }^{4}$. La plupart d'entre eux sont difficiles à dater; il semble que leur

- Premier article : Gallia, XXIV, 1966, p. 189-215.

(1) W. Fechs, Der Schiffsfund von Mahdia, Bilderhefle des deutschen archäologischen Instituls, Rom, 1962.

(2) Ch. Picaro, Musees et sites de l'Afrique du Nord, dans Revue archéologique, 1947, p. 221 et s.

(3) J. Schwartz, Sur des prélendus isiatiques, dans Latomus, 22, 1963, p. 472 et s.

(4) C.-C. Edgar, Calalogue général des Anliquilés du Musee du Caire, pl. V et s. ; P. Perdrift, Bronzes d'Egyple de la collection Fouquel, pl. XXIII et s. 
production soit due à une inspiration gréco-égyptienne ou gréco-orientale ${ }^{5}$ mais que la plupart des objets ainsi dénommés aient été fabriqués à l'époque romaine jusqu'à une date assez tardive. Ainsi se pose le problème des ateliers d'où sortirent certains d'entre eux, qui se rencontrent en nombre important dans la périphéric du monde romain, par exemple en Gaule ou en Europe centrale ${ }^{6}$.

Parmi les 15 objets étudiés, 4 ont été trouvés en Gaule de façon certaine ( $\mathrm{n}^{\text {os }}$ d'analyse : 80, 87, 122, 125). Les autres proviennent de collections particulières et ont toutes chances d'être d'origine plus lointaine : pour la plupart d'entre eux, les achats ont dû être effectués en Italic. Ils permettent toutefois une comparaison utile et nécessaire (voir les tableaux p. 159 et les figures 2 à 16 ).

Ils sont, pour la majorité, traités en creux (vases à parfum, lampes, appliques), ct nous signalerons que certaines particularités de composition s'expliquent par des procédés de fabrication correspondant à leur nature. Cependant on observe, pour l'un et l'autre groupe d'objets (massifs et en creux), les mêmes caractères dominants qui les distinguent nettement des objets de tradition gréco-romaine étudiés jusqu'ici (bronzes de Lyon et de Vienne)?

Le premier de ces caractères spécifiques est la présence de zinc qui apparaît à des taux égaux ou supérieurs à $1 \%$ dans les compositions de 10 objets sur les 15 exemplaires analysés. Par ailleurs ces pourcentages de zinc ne dépassent pas $8 \%$. Dans le groupe rles statuettes et statues de tradition romaine de Lyon et de Vienne, scules trois d'entre elles avaient des pourcentages comparables de zinc $^{8}$, sur plus d'une cinquantaine d'objets ${ }^{9}$. Nous ne tenons pas compte ici des cinq analyses mentionnées plus bas sous la rubrique "bronzes galloromains ", qui appartiennent du point de vue religieux à un groupe différent.

Deux hypothèses simples peuvent être formulées, qui expliqueraient la présence de ces pourcentages de zinc, curieusement faibles : le zinc peut provenir d'une refonte d'objets en laiton, mêlés à des bronzes de récupération ou à du cuivre vierge, éventuellement avec apport d'étain ; - le zinc peut être une impuretée ${ }^{10}$ caractéristique du cuivre utilisé avec lequel il constitue en quelque sorte un alliage naturel.

La seconde hypothèse paraîtrait quelque peu renforcée par la présence dans certains exemplaires d'un taux global d'impuretés (zinc non compris) particulièrement élevé. Indiquons, à titre d'exemple, que les pourcentages de nickel ${ }^{11}$ des exemplaires 120, 122 et 123 sont de l'ordre de $0,5 \%$ alors que dans le groupe des statuettes de tradition gréco-

(5) A. Adriavi, Microasialici o alessandrini i grolleschi di Mahdia, Römische Milleilungen, 1963, p. 80 et s. ; W. Fechs, op. 1 .

(6) J. SCHWARTZ, op. $l$.

(7) M. PICon, S. Boccin: , J. Coxpamin, Recherches techniques sur des bronzes de Gaule romaine, Gallia, XXIV; 1966 , p. 189 et s.

(8) Trois autres etaient des laitons vrais : hauts pourcentages de zinc, et faibles pourcentages d'étain.

(9) Nous avons été amenés à éliminer l'objet de style alexandrin $n^{\circ} 45$, à la suite d'une étude que nous avons effectuée sur la composition de sa patine, et du fait de certains de ses caractères stylistiques (cf. S. Botchi: $A$ propos d'un grolesque alexandrin, à paraître dans les Mélanges de l'École française de Rome).

(10) Nous conservons le terme d'impureté sans vouloir exclure par là toute idée d'une recherche intentionnelle des minerais.

(11) L.e nickel est en principe une impureté du cuivre, il n'est donc pas directement lié à la présence de zinc. Ce dernier peut d'ailleurs être complètement éliminé au cours des traitements nécessaires à l'élaboration du métal, s'ils sont conduits avec soin, et cela assez facilement. Le nickel, par contre, s'élimine moins aisément. 
romaine de Lyon et de Vienne, pas un seul exemplaire n'en contient en proportions comparables $^{12}$ : presque toutes en présentent moins de $0,1 \%{ }^{13}$. Ajoutons que l'on connait bien, particulièrement à la suile des nombreux travaux de E. R. Caley ${ }^{14}$, la pureté des cuivres utilisés dans le monde romain pour les émission monétaires jusqu'à la seconde moitié du ${ }^{11}{ }^{\mathrm{e}}$ siècle et que les taux de nickel y sont toujours fort inférieurs à $0,1 \%$. Cette absence presque complète du nickel dans les divers alliages à base de cuivre dont sont constitués les objets romains semble donc devoir interdire l'hypothèse d'une refusion pour expliquer les bas titres de zinc des statuettes où le nickel est important. Pour ces exemplaires où l'hypothèse d'un alliage naturel peut ètre envisagée ${ }^{15}$, on penserait volontiers à une technique métallurgique restant en marge du monde romain, où l'affinage du métal serait moins poussé qu'ailleurs. Et comment ne pas songer, dans ces conditions, au district rhénan ${ }^{16}$, parmi tous ceux qui sont susceptibles d'être rangés dans le cadre ainsi défini ?

Cependant, si certains de ces objets ont été trouvés en Gaule et nous intéressent plus particulièrement, la plupart de ceux qui nous ont servi d'éléments comparatifs ont sans nul doute une origine étrangère, peut-être fort lointaine. Il est incontestable alors qu'on ne peut étendre l'explication proposée à la totalité des objets, une bonne partie de ceux-ci ayant de plus des proportions d'impuretés qui ne s'écartent pas sensiblement des valeurs moyennes observées sur les bronzes romains. Il semble donc que, dans le problème de la présence du zinc en faible proportion dans les bronzes de type alexandrin, on ne puisse faire abstraction, pour bien des exemplaires, de l'hypothèse d'une refusion, voire mème d'une cémentation restreinte, peut-être à l'état liquide ${ }^{17}$. Il existe donc finalement trois types d'alliages à bas titre de zinc entre lesquels il est à peu près impossible de décider en l'absence d'impuretés notables. Or si de tels alliages paraissent rares parmi les bronzes statuaires, ils sont assez communs pour la vaisselle de bronze (certaines de nos statuettes s'y rattachent par plus d'un (aractère). Nous en avons nous-même retrouvé trois exemplaires en poursuivant nos

(12) Au contraire, dans le domaine incomparablement plus reduit des statues de grandes dimensions que nous avons étudiées, trois objets, dont la statue de Pacatianus, contiennent des proportions de nickel de l'ordre de $0,5 \%$. On peut se demander si cette absence dans le groupe des statuettes, et cette présence dans celui des statues ne seraient pas a mettre en relation avec une fabrication locale (contra: A. Fraxci-I,axord, Monumenls Piol, 51, 1958, La slalue de bronze reconstiluée dile de Pacalianus all Musée de Vienne (1sìre), p. 93 et s.).

(13) L'ensemble des observations faites sur la présence du nickel dans les bronzes ne permet pas d'accepter l'interprétation de M. J. Maréchal (article Métallurgie du Jiclionnaire des Terhniques, II, éd. de l'Accueil, Paris, 1964), selon qui la présence de cet élément serait intentionnelle dans les bronzes au plomb, et destinée à faciliter la répartition du plomb afin d'éviter les ségrégations.

(14) Voir en particulier E.R. Cat.ey et H. D. Mc Brave, Chemical composilion of antoniniani of Trajan Decius, Trebonianus Gallus, and Valerian, The Ohio Journal of Sciences, 56 (5), 1956, p. 285-289; E. R. CAs.EY, Orichalcum and relaled ancienl alloys, Numismalic Notes and Monographs, n 151, The Americun Numismalic Sociely, New York, 1964.

(15) Le cas du no 122 dépourvu de zinc ne peut être systématiquement disjoint des deux autres. Nous avons signalé (note 5) que le nickel l'emporte sur le zinc dans la caractérisation d'un type de cuivre. Cela résulte de la très grande volatilité du zinc.

(16) On peut signaler la proportion élevée d'objets contenant du zinc parmi la vaisselle de bronze du Musée de Nimegue: M. H. P. DEx Boestero, Descriplion of the collections in the Rijlsmuseum G. M. Kam at Nijmegen, V, The Bronze Vessels, 1956, Speclrochemical analyses, 1966. I.es vases plastiques du même Musée présentent des taux de zinc comparables à ceux de nos exemplaires. Ils sont de provenance locale. En dehors du district rhénan, il faudrait orienter les recherches vers les centres metallurgiques de l'burope centrale : la présence du nickel y est un caractère fréquent à l'Age du bronze.

(17) C. E. Salix, La Civilisalion mérubingienne, III, p. 1.10-141, Paris, 1957. 
recherches sur les patères tournées ( $\mathrm{n}^{0 \mathrm{~s}} 107,113$ et 114), exemplaires qui s'ajoutent au vase $n^{0} 10$ déjà étudié. Mais l'existence même de ces alliages, leur relative abondance en dehors du domaine romain, leur prédominance parmi les bronzes de type alexandrin paraissent indiquer au moins que le seul point commun des fabrications de ce dernier type est sans doute la persistance plus ou moins atténuée d'une industrie qui devait à l'origine s'être spécialisée dans les objets en laiton ${ }^{18}$. C'est dire à quel point il serait fondamental de connaitre non sculement les objets de type alexandrin provenant de l'Orient méditerranéen, mais plus encore d'acquérir un minimum de connaissances sur la structure, la spécialisation et l'importance des ateliers de bronziers de l'Antiquité. Dans une autre voie, il faudrait s'assurer de la provenance des alliages caractérisés par l'association du zinc en impureté avec des traces importantes de nickel, recherche qui nous conduirait tout naturellement en direction du district métallurgique rhénan et en Europe centrale.

Les pourcentages de plomb et d'étain n'offrent pas de caractères très notables. Les taux d'étain particulièrement élevés de certains exemplaires (voir le diagramme, fig. 1) doivent être imputés aux techniques bien connues des bronzes à patères, ou bronzes coulés minces, déjà étudiés ${ }^{19}$. La technique de ces vases plastiques et de ces vases à reliefs n'est donc pas essentiellement différente de celle des vases coulés ou tournés. On peut se demander, étant donné la présence de faibles taux de zinc, bien moins fréquente dans les statuettes romaines que dans le domaine de la vaisselle, s'il ne conviendrait pas de rapprocher les productions de type alexandrin de cette dernière. Ajoutons enfin, à l'appui de cette hypothèse, que deux petits objets massifs (n ${ }^{08} 124$ et 125) présentent une composition qui est celle de la vaisselle coulée et tournée.

\section{BHONZES IDE TYPE GALLO-ROMAIN ET GAULOIS}

Les bronzes gallo-romains étudiés se réfèrent à la religion celtique, puisque ce sont tous les cinq des Dispaler; ils doivent être rattachés par leur aspect esthétique à l'art romain : le sens des proportions, l'équilibre, le classicisme des traits les rendent dépendants des conceptions méditerranéennes (voir le tableau p. 160 et les figures 17 â 21 ).

Les caractères propres à la composition de ce groupe sont ici encore la présence de zinc à de faibles concentrations et, pour certains exemplaires, le nickel.

Les pourcentages de zinc n'excèdent pas $6 \%$, mais si 3 statuettes ont des taux égaux ou supérieurs à $1 \%$, les deux autres ont respectivement $0,5 \%$ et $0,1 \%$. Trois exemplaires ont des taux de nickel de l'ordre de 0,5\% (n ${ }^{08} 128,130$ et 132) et, pour l'ensemble, le taux global d'impuretés reste assez élevé. On peut donc admettre raisonnablement l'interprétation proposée pour quelques-uns des bronzes alexandrins où les taux d'impuretés suggéraient l'emploi d'un cuivre local (ou plutôt régional). On peut à ce propos rappeler que si les

(18) Ce caractère intentionnel des traces de zinc apparaît d'une manière assez nette sur les exemplaires 117 et 118 dont les pourcentages d'étain sont les plus bas parmi les bronzes coulés minces. Mais la plupart des autres objets montrent des pourcentages de zinc trop faibles pour qu'il existe d'autre alternative qu'une localisation géographique très stricte (qui ne paraît pas pouvoir être soutenue pour les raisons que nous avons données), ou une tradition de fabrication.

(19) C.t. Gallia, art. cit. 

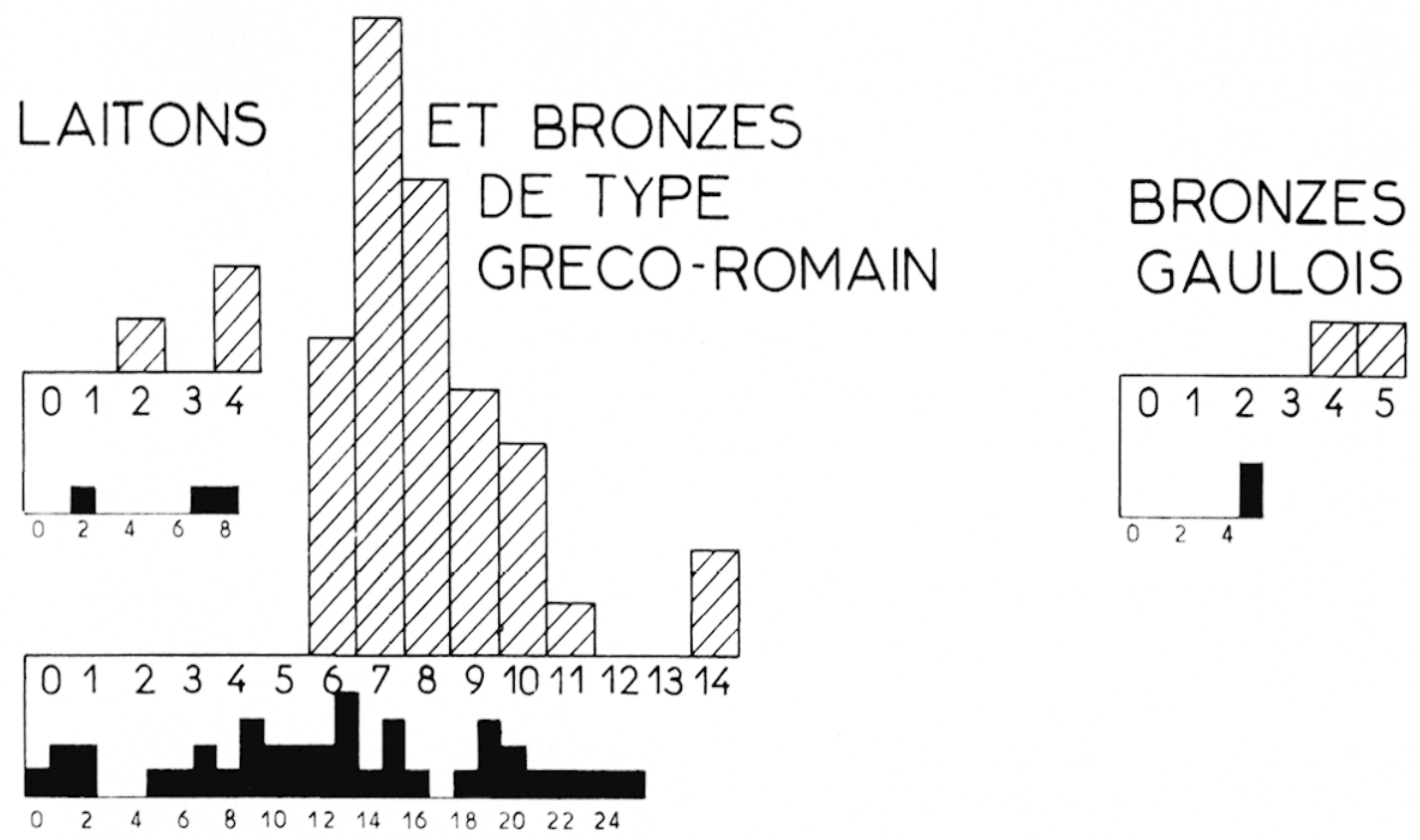

BRONZES DE TYPE $\oslash$ ALEXANDRIN

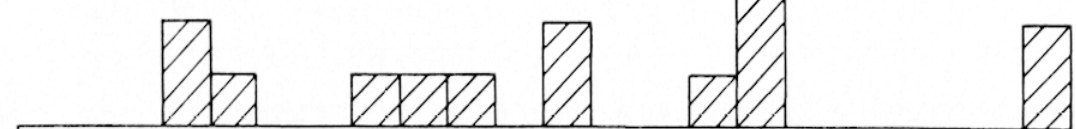

0123456789101112131415161718192021
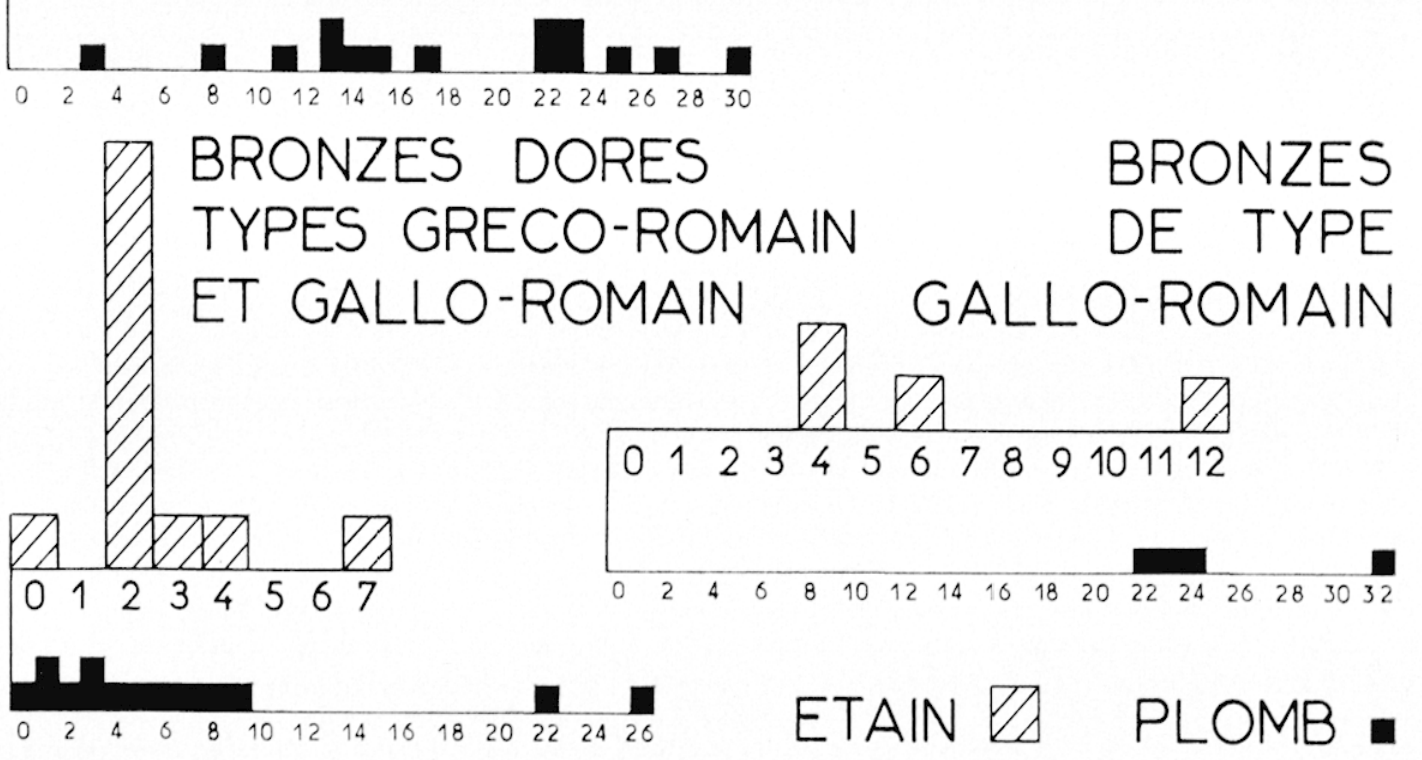

1 Pourcentages d'étain et de plomb dans les bronzes statuaires. Le nombre d'échantillons correspondant à chaque pourcentage d'étain et de plomb est figuré verticalement par les hauteurs hachurées ( $\mathrm{Sn}$ ), ou en noir ( $\mathrm{Pb})$. 
statuettes de tradition gréco-romaine des Musées de Lyon et de Vienne ne nous ont fourni aucun alliage de ce type, nous en avons trouvé trois parmi les quelques grandes statues de res mêmes villes, étudiées jusqu'ici². On peut donc penser qu'il existe très probablement entre les diverses statuettes de ce groupe une relation créée par une origine commune du cuivre employé, qu'il s'agisse des produits d'ateliers installés au voisinage même des lieux de production, ou plus simplement situés dans la zone de diffusion de ces produits. Là aussi des investigations poussées dans le district rhénan et dans les centres métallurgiques de l'Europe centrale pourraient être particulièrement fructueuses.

Il importe de souligner que ces statuettes qualifiées par nous de gallo-romaines appartiennent d'un point de vue strictement technique au monde romain ${ }^{21}$. Les très hauts pourcentages de plomb associés à des pourcentages d'étain généralement bas (voir le diagramme, fig. 1) sont à cet égard très significatifs, et doivent être interprétés comme le résultat d'une assimilation complète des techniques de la statuaire romaine. Il est même très probable que ces taux de plomb particulièrement élevés puissent être mis en relation avec la technique d'une époque assez tardive. La technique romaine se révèle encore dans la composition de l'exemplaire no 128 qui est doré et possède de ce fait un taux d'étain qui est le plus faible de tous, comme c'est alors de rigueur pour la dorure par placage sur rayures ${ }^{22}$.

Ajoutons, afin de bien comprendre l'évolution du bronze en Gaule depuis la conquète jusqu'à l'époque des statuettes gallo-romaines précédentes, que l'étude systématique de fabrications grauloises et gallo-romaines précoces nous a montré que la technique gauloise du bronze n'avait pratiquement pas évolué depuis la fin de l'Age du bronze jusqu'à l'époque romaine. Les auvres de la fin de l'Indépendance ou de peu postérieures à celle-ci, appartiennent encore à ce mondle. Nous en signalerons deux, assez significatives de cette période, en attendant une publication développée des résultats qui la concernent. L'une est la tète d'un dieu gaulois, trouvée près de Rouen, apparentée au dieu de Bouray ${ }^{23}$; l'autre est une protomé de taureau à cornes bouletées ${ }^{24}$, du Musée de Vienne. Pour l'un et l'autre objet

(20) Parmi celles-ci, relevons le cas du no 39 qui possède $4 \%$ de zinc.

(21) Nous ne tenons pas compte ici des quelques caractères déduits de la présence des impuretís qui pourraient témoigner d'une technique d'élaboration du métal à partir de ses minerais, peut-être un peu moins évoluée que dans l'ensemble du monde romain. D'ailleurs il est fort probable que les ateliers de fabrication des statuettes aient éte plus ou moins indépendants des centres de production du métal.

(22) In certain nombre d'analyses nouvelles de bronzes dorés ont été faites et rassemblées dans les tableaux en fin d'article. Si l'on y ajoute celles qui ont été mentionnées dans le précédent article, on peut dés à présent noter les caractires suivants. Le taux d'etain particulièrement faible de l'ensemble doit être mis en relation avec la facilité conférée par un support malléable à l'opération de placage de la feuille d'or, ainsi que nous l'avions déjà signalé (Gallia, XXIV, 1966, p. 208). Cette condition, bien que très généralement respectée, ne devait cependant pas être impérative. On connaît l'exemple célèbre de la statue de Lysippe dont la dorure malencontreuse avait été ordonnée par Néron. (Pi.s., Misl. Nal. XXXIV, 19, 63). Le cas du no 140 représente peut-être un exemple du même genre, où la dorure aurait pu ne pas avoir été prévue initialement. Yais le caractère le plus intéressant que viennent confirmer ces nouvelles analyses de bronzes dorés, c'est la proportion élevée des bronzes à faible pourcentage de plomb. Il nous semble que le roile attribué au plomb dans la dorure antique soit inexact, et que la technique par placage sur rayures se soit adaptée, peut-être tardivement, à des alliages tres riches en plomb, comme elle l'a fait, ici ou là, pour des alliages plus riches in itain. Les études techniques des procédés de dorure antiques feront l'objet d'une publication séparéc.

(23) R. Lantier, Monuments Piol, 34, 1934, p. 35 et s.

(24) J. Dírintserts, Manuel d'archéologie préhislorique, cellique el gallo-romaine, IV, p. 1018 el s., fig. $691,1$. 
les taux de plomb restent très faibles. Ce n'est que progressivement que les ateliers locaux s'essaieront à la technique des bronzes au plomb, depuis longtemps florissante en Italic en même temps que leurs habitudes de travail se modifieront. L'exemplaire de Vienne a de plus des traces d'antimoine assez fortes, de l'ordre de $0,5 \%$. Celles-ci témoignent, au travers des refontes, du courant d'importation en provenance d'Europe centrale, qui avec le courant italique a submergé l'industrie locale du bronze au temps de l'indépendance gauloise.

I'ATĖRES (suile au lableau publié dans (Gallia, XXIV, 1966, p. 212).

\begin{tabular}{|c|c|c|c|c|c|c|c|}
\hline \multirow{2}{*}{$\begin{array}{l}\text { Numéro } \\
\text { D'ANalyse }\end{array}$} & \multicolumn{4}{|c|}{ Composition } & \multirow{2}{*}{$\begin{array}{l}\text { ÉpAISSEUR } \\
\text { en mm }\end{array}$} & \multirow{2}{*}{ Structures } & \multirow{2}{*}{ Désignation } \\
\hline & $\mathrm{Cu}$ & Sn & $\mathrm{Pb}$ & $\mathrm{Zn}$ & & & \\
\hline 107 & 80 & 6 & 8 & 6 & 0,8 & C & $\mathrm{F}$ \\
\hline 108 & 64 & 6 & 30 & & 1,2 & C & $\mathrm{F}$ \\
\hline 109 & 90 & 10 & & & 1,5 & C & $\mathrm{F}$ \\
\hline 110 & 89 & 11 & & & 0,6 & $\mathrm{R}$ & F \\
\hline 111 & 88 & 12 & & & 0,5 & $\mathrm{R}$ & F \\
\hline 112 & 87 & 13 & & & 0,8 & $\mathrm{R}$ & F \\
\hline 113 & 67 & 15 & 13 & 5 & 0,6 & C & $\mathrm{F}$ \\
\hline 114 & 59 & 17 & 19 & 5 & 0,8 & C & $\mathrm{F}$ \\
\hline
\end{tabular}

BRONZES "ALEXANIDRINS"

\begin{tabular}{|c|c|c|c|c|c|}
\hline \multirow{2}{*}{$\begin{array}{l}\text { Numéro } \\
\text { D'analyse }\end{array}$} & \multicolumn{4}{|c|}{ Composition } & \multirow{2}{*}{ Désignation } \\
\hline & $\mathrm{Cu}$ & Sn & $\mathrm{Pb}$ & $\mathrm{Zn}$ & \\
\hline 115 & 92 & 3 & 3 & 2 & Lyon. Vieillard grotesque (A 2002) (fig. 2) \\
\hline 116 & 82 & 3 & 11 & 4 & Lyon. Myrmidon (H 1184) (fig. 3) \\
\hline 117 & 76 & 4 & 13 & 7 & $\begin{array}{l}\text { Lyon. Lampe en forme de tète de nègre } \\
\text { (E } 413 \text { ) (fig. } 4 \text { ) }\end{array}$ \\
\hline 118 & 57 & 7 & 30 & 6 & Lyon. Vase plastique (E 227) (fig. 5) \\
\hline 80 & 76 & 8 & 15 & 1 & V. Vieillard grotesque (V. 38) (fig. 6) \\
\hline 87 & 69 & 9 & 14 & 8 & F. Grotesque (A 2473) (fig. 7) \\
\hline 119 & 66 & 11 & 23 & - & Lyon. Vase plastique (E 227) (fig. 8) \\
\hline 120 & 65 & 11 & 23 & 1 & Lyon. Vase plastique (E 408) (fig. 9) \\
\hline 121 & 61 & 14 & 22 & 3 & Lyon. Vase plastique (A 2127) (fig. 10) \\
\hline 122 & 67 & 15 & 13 & 5 & F. Buste d'«isiaque?» (1967-1) (fig. 11) \\
\hline 123 & 63 & 15 & 22 & & Lyon. Vase plastique (E 330) (fig. 12) \\
\hline 124 & 60 & 15 & 25 & & Lyon. Esclave (E 408) (fig. 13) \\
\hline 125 & 58 & 15 & 27 & - & F. Grotesque (A 2000) (fig. 14) \\
\hline 126 & 71 & 21 & 8 & & Lyon. Vase à reliefs (L 240) (fig. 15) \\
\hline 127 & 58 & 21 & 17 & 4 & Lyon. Vase plastique (L 1348) (fig. 16) \\
\hline
\end{tabular}


BRONZES GAULOIS ET GALLO-ROMAINS

\begin{tabular}{|c|c|c|c|c|c|}
\hline \multirow{2}{*}{$\begin{array}{l}\text { NuMÉRO } \\
\text { D'ANALYSE }\end{array}$} & \multicolumn{4}{|c|}{ Composition } & \multirow{2}{*}{ Désignation } \\
\hline & $\mathrm{Cu}$ & Sn & $\mathrm{Pb}$ & $\mathrm{Zn}$ & \\
\hline 128 & 85 & 2 & 7 & 6 & F. Dispater (A 1880) (fig. 17) \\
\hline 129 & 72 & 4 & 24 & - & F. Dispater (A 1881) (fig. 18) \\
\hline 130 & 72 & 4 & 23 & 1 & F. Dispater (E 489) (fig. 19) \\
\hline 131 & 68 & 6 & 22 & 4 & F. Dispater (L 10) (fig. 20) \\
\hline 132 & 56 & 12 & 32 & - & F. Dispater (L 21) (fig. 21) \\
\hline 133 & 91 & 4 & 5 & & F. Tête de Gaulois (E 377) (fig. 22) \\
\hline 134 & 90 & 5 & 5 & & V. Taureau à cornes bouletées (V 74) (fig. 23$)$ \\
\hline
\end{tabular}

BRONZES DORÉS (voir ci-dessus, p. 158, note 22)

\begin{tabular}{|c|rrrr|l|}
\hline \hline 39 & 96 & - & - & 4 & F. Aviron (manche) (1967-5) \\
40 & 97 & 2 & 1 & - & F. Aviron (pale) (1967-5) \\
135 & 97 & 2 & 1 & - & F. Couronne (1967-2) \\
136 & 95 & 2 & 3 & - & F. Patte de cheval (1967-3) \\
137 & 95 & 2 & 3 & - & V. Bouclier décoratif ( (V 908) \\
41 & 94 & 2 & 4 & - & V. Dauphin (placage) (V 83) \\
42 & 90 & 2 & 8 & - & V. Dauphin (placage) (V 83) \\
43 & 76 & 2 & 22 & - & F. Patte d'animal (1967-4) \\
138 & 92 & 3 & 5 & - & Besançon. Trompe d'éléphant. \\
139 & 94 & 4 & 2 & - & Besançon. Queue de bovidé. \\
140 & 67 & 7 & 26 & - & Besançon. Pied. \\
Besançon : la trompe d'éléphant et le pied humain proviennent de Besançon mème ; la queue \\
de bovidé a été trouvée à Mandeure. \\
\hline
\end{tabular}

$V$. désigne le Musée de Vienne, $F$. le Musée de Fourvières à Lyon, Lyon, le Palais des Beaux-Arts à Lyon. Ces indications n'ont aucun rapport avec l'origine des objets. Seuls le vieillard de Vienne (80), les deux grotesques : (87) provenant de Nyons, (125) provenant d'Arles, le buste d'« isiaque " (122: Lyon), ont une origine déterminée. Les numéros sont ceux des inventaires, sauf pour Vienne où ils se rapportent au catalogue des bronzes de ce Musée. Pour les patères, par suite de leur état fragmentaire, il n'a pas été donné de numéro autre que celui de l'analyse.

Maurice Picon, Jeanne Condamin, Stéphanic Boucher. 


\section{APPENDICE}

\section{Pline l'Ancien, Histoire naturelle, XXXIV : plumbum argenlarium}

Les termes plumbum argentarium employés par Pline au livre XXXIV ont donné lieu à diverses interprétations. Les indications fournies par cet auteur sont les suivantes :

10 Bronze de Campanie, fabriqué à Capoue $(\mathrm{XX}, 95)$. - A 100 livres de cuivre sont ajoutées 10 livres de plumbum argentarium $(=9,1 \%$ de plumbum argentarium).

$2^{\circ}$ Aulres régions d'Ilalie (XX, 96). - A 100 livres de cuivre sont ajoutées 8 livres de plumbum argenlarium $(=7,4 \%)$.

$3^{0}$ Alliage pour les stalues el les tables (XX, 97). — Au lingot de métal (cuivre) est ajouté $1 / 3$ de "cuivre» de récupération. Pour cent livres de ce métal, on utilise 12 livres $1 / 2$ de plumbum argenlarium. Le "cuivre" de récupération peut être presque pur ; mais ce peut être déjà un alliage. La proportion de plumbum argentarium variera donc de 11 à $13 \%$ environ.

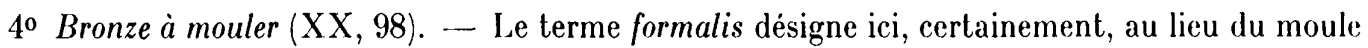
dans lequel on coule l'objet, l'ébauche coulée (contra: II. Le Bonniec, Pline l'Ancien, Histoire nalurelle, XXXIV, Paris, Les Belles Lettres, ad loc., qui s'inspire avec les autres traducteurs du commentaire d'Hardouin : "ad formas faciendas»). On ne connaît guère de moules de bronze à l'époque romaine, et il serait étonnant que Pline ne mentionnât pas dans son traité le bronze moulé. A 100 livres de cuivre, on ajoute 10 livres de plumbum nigrum et 5 livres de plumbum argenlarium, soit une proportion de $8,7 \%$ de plumbum nigrum, et de $4,4 \%$ de plumbum argenlarium.

$5^{0}$ Bronze à marmiles (XX, 98). - A 100 livres de cuivre, on ajoute 3 à 4 livres de plumbum argentarium, soit 2,9 à $3,8 \%$.

M. H. Le Bonniec traduit plumbum argenlarium par plomb argentifère, reprenant ainsi l'interprélation de Blümner ${ }^{25}$. Cette traduction est impossible pour plusieurs raisons. Tout d'abord, rien ne prouve qu'argentarium signifie ici "argentifère "; cette interprétation est due à un parallélisme abusif entre le terme français et le terme latin. Dans le Thesaurus Linguae latinae, Otto donne d argentarius, en référence au texte de Pline, la signification suivante : "argento similis propler colorem ». D'autre part, Pline précise que c'est du plumbum nigrum (plomb noir, notre plomb actuel) qu'est extrait l'argent (XLVII, 158), et il mentionne en XX, 98 un alliage où entrent plumbum nigrum et plumbum argentarium, dissociant ainsi nettement les deux métaux. Enfin, on s'étonne que Pline ait ignoré que les bronzes contenaient de l'étain.

M. K. G. Bailey ${ }^{26}$ avait donné un autre sens à argentarium. Pline, en effet (XLVIII, 160), parle d'un métal constitué pour moitié de plomb blanc (étain) et pour l'autre moitié de plomb noir (plomb), qu'il nomme argenlarium. Il est alors tentant d'interpréter de cette façon les termes plumbum argentarium dans les passages qui concernent les différents alliages du bronze. Cependant M. H. Le Bonniec remarque à juste titre que le plumbum argentarium de ces alliages, qualifié d'Hispaniensis (XX, 95) ne peut être guère confondu avec l'argentarium, alliage de plomb et d'étain, signalé par Pline comme l'une des contrefaçons du slagnum (XLVIII, 160), qu'on n'avait aucune raison d'aller chercher sous cette forme en Espagne. L'expression de Pline "hoc nunc aliqui argentarium appellant" montre

(25) BlümNer, Technologie und Terminologie, IV, p. 181.

(26) M. K. C. BaILEy, The Elder Pliny's chapters in chemical subjecls, part II, London, 1932. 
eflectivement que cette appellation n'est pas courante, et qu'elle élail peut-itre récente à son époque. D'aulre part, cetle interprétation supposerail que fûl préparé à l'avance un alliage plomb-étain qui aurait été ajouté tel quel au cuivre ; ce procédé semble improbable.

Il faut remarquer que les noms donnés aux métaux dans ce texte sont des noms de couleur : plumbum nigrum, plumbum candidum ou album, aes candidum. Il semble bien qu'argentarium soil aussi un qualificatif qui double candidum ou album, et que le plumbum argenlarium soit tout simplement le plumbum album, c'est-a-dire l'étain. On comprend alors qu'on puisse introduire dans le même alliage du plumbum nigrum el du plumbum argenlarium, que le plumbum argentarium puisse cetre qualifié d'Ilispaniensis (Pline mentionne en eflet des mines de plumbum album en Espagne). On comprend aussi que se soit appelée argenlarium la contrefaçon d'un alliagre de teinte arquentée utilisé pour la fabrication des miroirs. Argenlarium ne désignerait donc que la couleur de certains métaux, et non leur nature.

Les analyses opérées sur les objets antiques appellent les constatations suivantes:

10 Bronze à marmiles. - - Il contient d'apres Pline 2.9 a $3,8 \%$ de plumbum argentarium. C'est effectivement la valeur moyenne de l'élain qu'on trouve pour les grosses pièces, seaux, chaudrons, vaisselle commune27. Techniquement, il est impossible que ces objets soient fails d'un alliage plomb-cuivre, du fait de la lechnique du martelage qu'ils supposent.

$2^{0}$ Bronze de Campanie. -..- La vaisselle de Campanie, ou dite telle, présente a l'analyse des taux d'étain compris entre 8 et $12 \%$. Beaucoup de ces objets sont martelés, ce qui interdit l'usage d'un alliage qui comprendrait $9 \%$ de plomb et pas d'étain (10) livres de plumbum argenlarium pour 100 de cuivre).

30 Bronze à mouler. .. Sa composition (5) livres de plumbum argenlarium el 10 livres de plumbum nigrum pour 100 de cuivre) correspond assez bien à celle de la multitude des objets romains contenant à la fois de l'étain el du plomb, qui sont obtenus par finissage d'une ébauche moulée (vaisselle, quincaillerie). Cette technique constitue, au début de notre ire, une révolution dans l'art de travailler le bronze (bronzes coulés minces) ${ }^{28}$.

$4^{0}$ Alliage pour stalues. - Dans ce domaine, il semble que les pourcentages de plumbum argentarium donnés par Pline soient très supérieurs aux pourcentages réels d'étain. Je plus, Pline ne mentionne pas la présence du plomb, qui est quasi-générale dans celle calégorie. Il est possible que l'auteur se réfère à la tradilion grecque, et que l'introduction du plomb, bien que pratiquée. par les bronziers, n'ait pas été portée clairement à la connaissance des utilisateurs. En outre, on manque de données certaines sur les grandes statues du $1^{\mathrm{er}}$ siècle apris. J.-C. et il est probable que l'introduction du plomb se soit faite progressivement ${ }^{29}$.

(27) Maria H. P. den Boestern, Descriplion of the collections in the Rijlismuseum G. M. Kam at Nijmegen, V, The Bronze Vessels, 1956, Spectrochimical analyses of the Bronze Vessels, 1966.

(28) M. Picon, S. Bouchir, J. Coxidam, Recherches techniques sur des bronzes de Gaule romaine, Gallia, XXIV, 1966, p. 204.

(29) V. les exemplaires de Vienue et de Lyon, Gallia, l. c. 


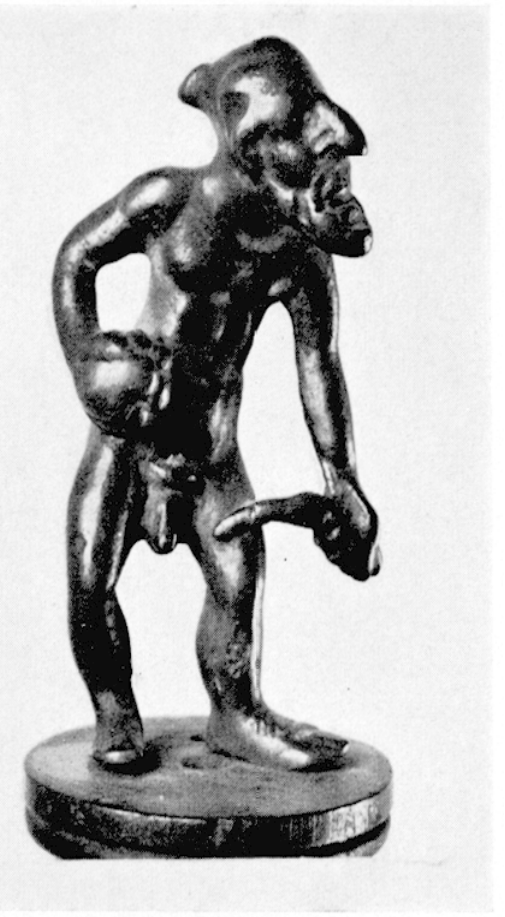

2 Vieillard grotesque. Lyon, Palais des Beaux-Arts.



I Lampe. Lyon, Palais des Beaux-Arts.

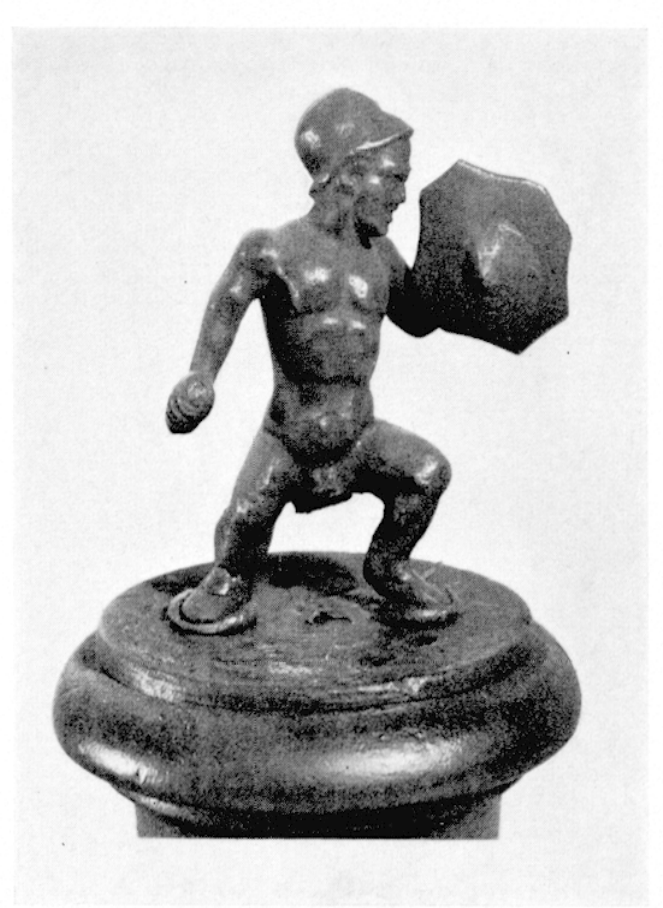

3 Myrmidon. Lyon, Palais des Beaux-Arts.

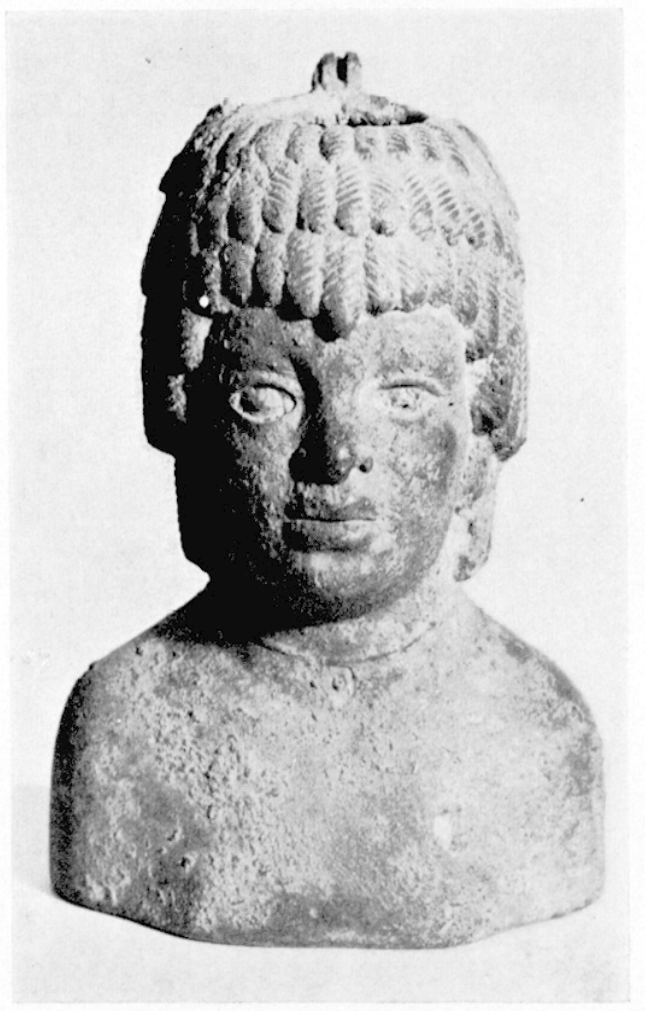

5 Vase plastique. Lyon, Palais des Beaux-Arts. 


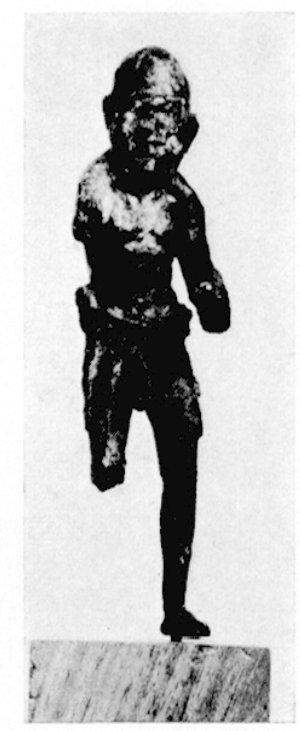

6 Vieillard grotesque. Musée de Vienne.

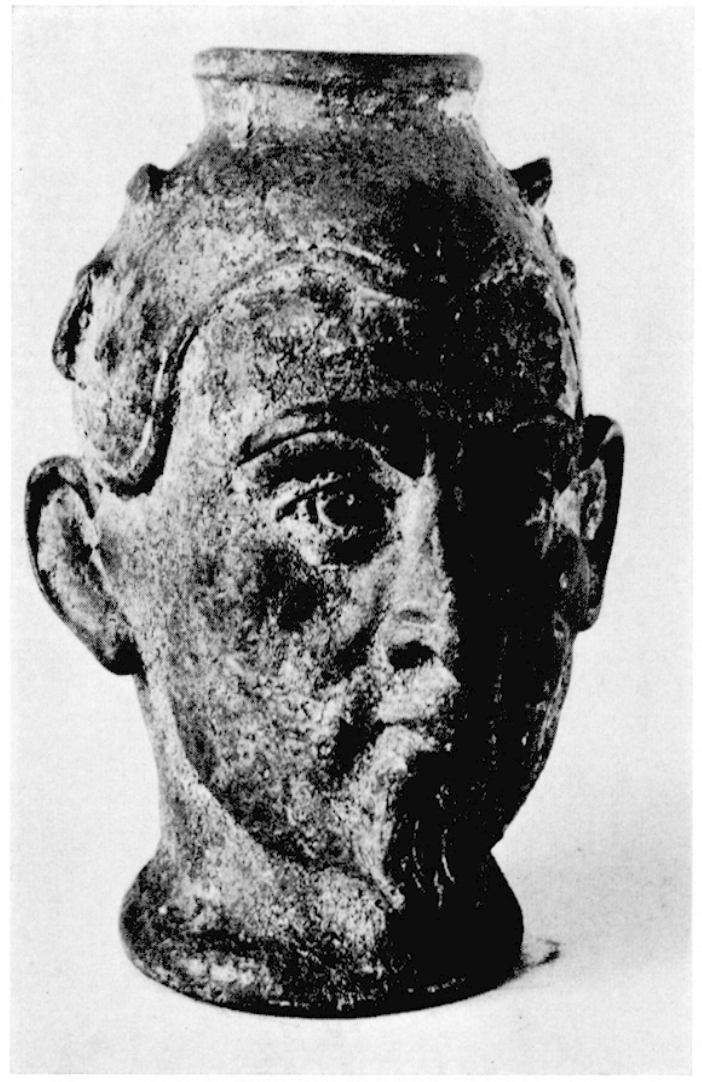

9 Vase plastique. Lyon, Palais des Beaux-Arts.

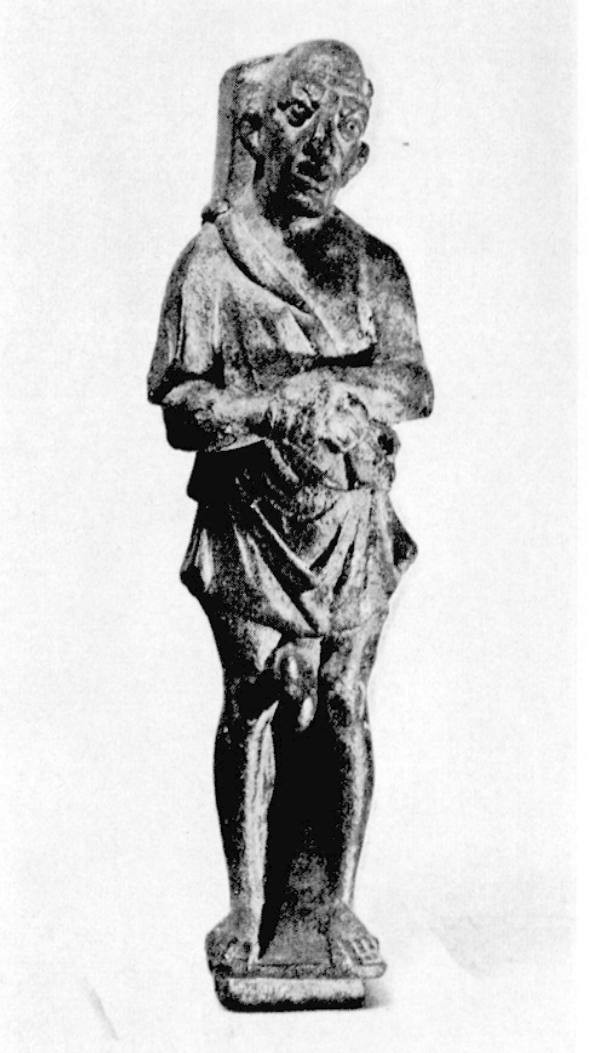

7 Grotesque. Lyon, Musée de Fourvières.

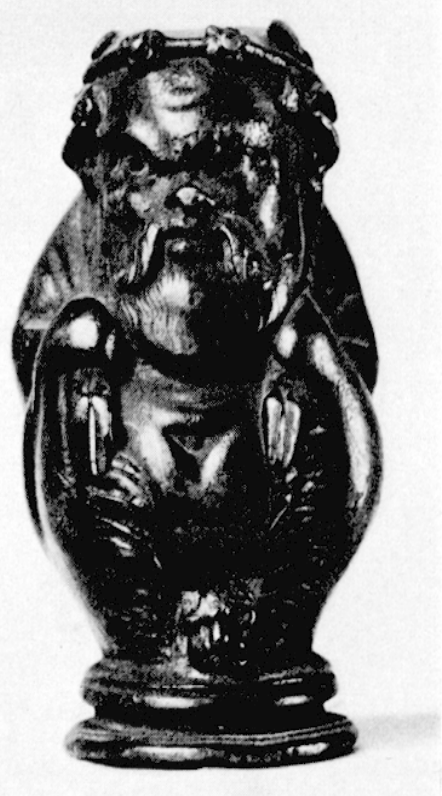

8 Vase plastique. Lyon, Palais des Beaux-Arts. 


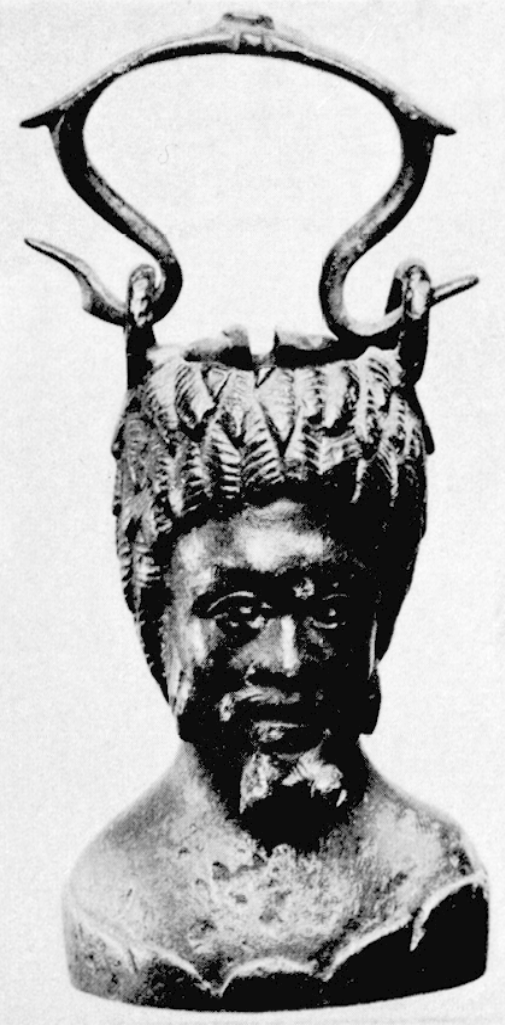

10 Vase plastique. Lyon, Palais des Beaux- $\Lambda$ rts.

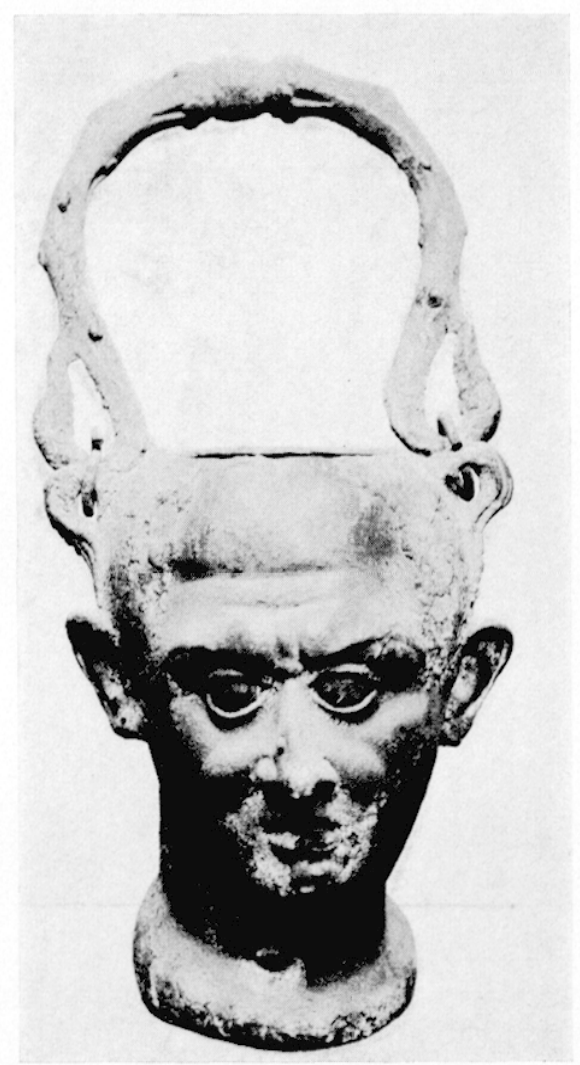

1:2 Vase plastique. Lyon, Palais des Beaux-Arts.

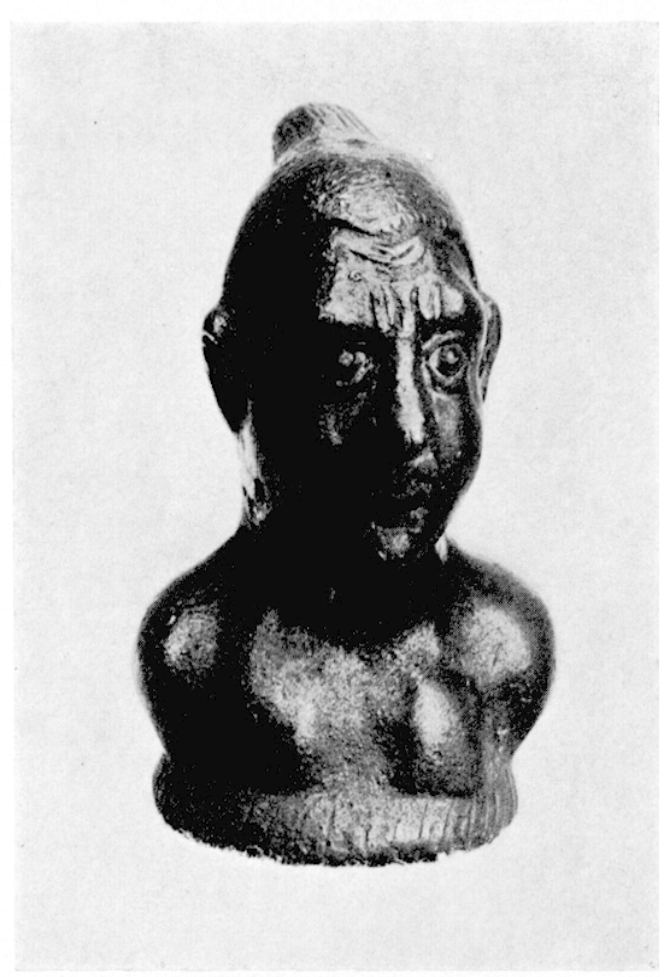

11 Buste d' "isiaque ". Lyon, Musée de Fourvières.

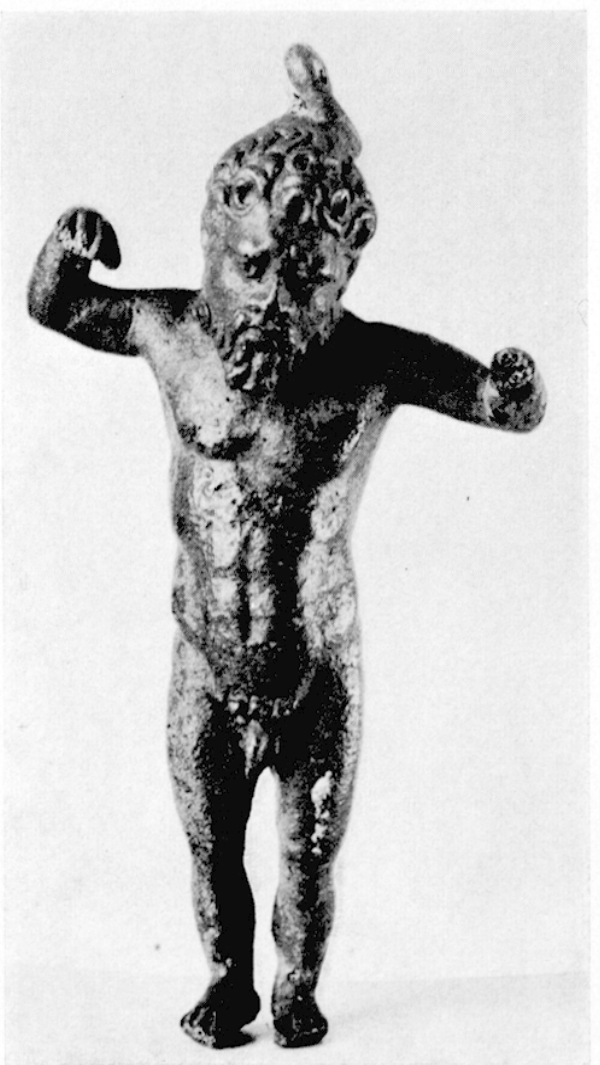

I3 Esclave. Lyon, Palais des Beaux-Arts. 

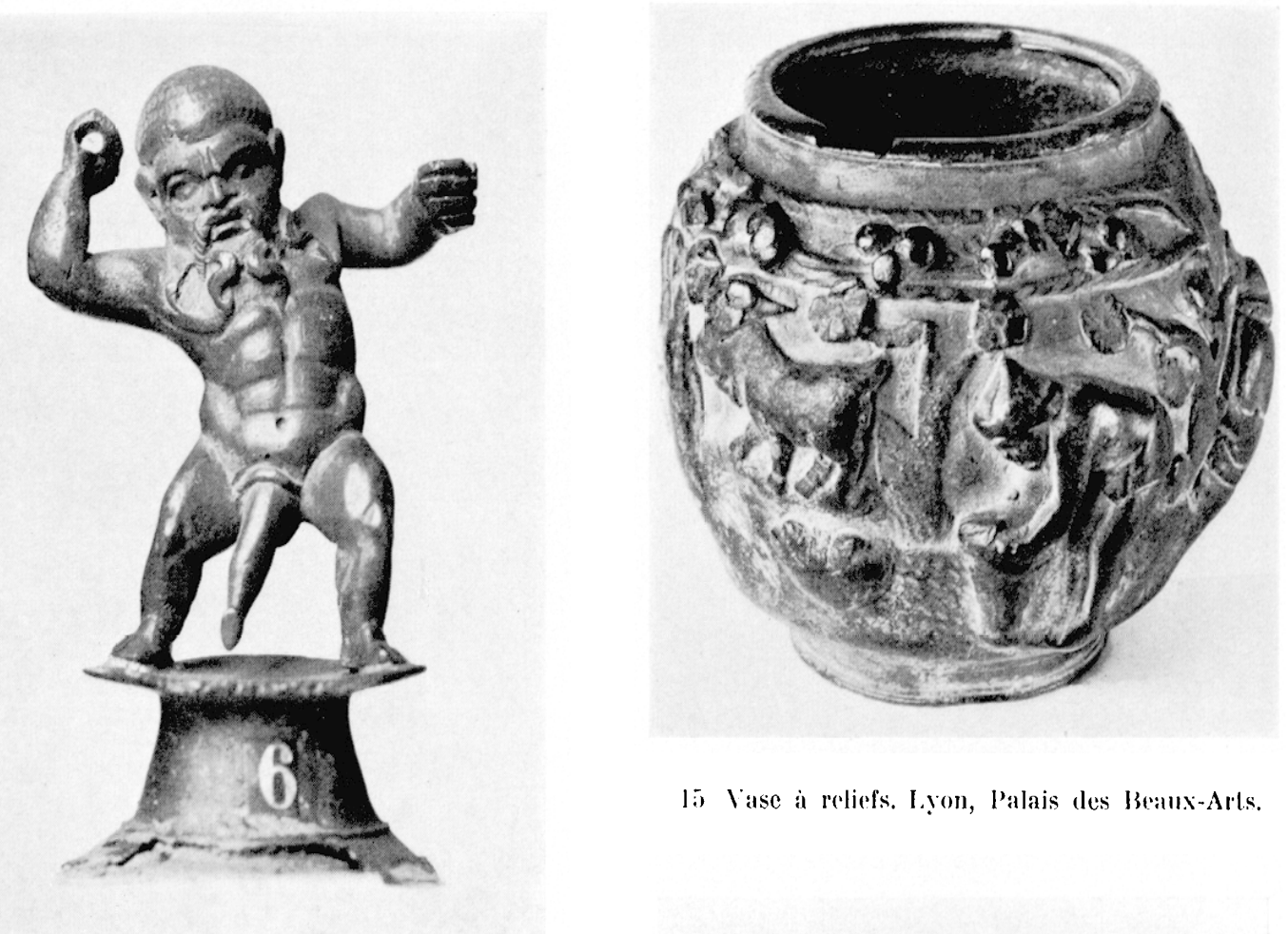

15) Vase à reliefs. Lyon, Palais des Beaux-Arts.

14 Grotesque. Lyon, llusie de liourvières.

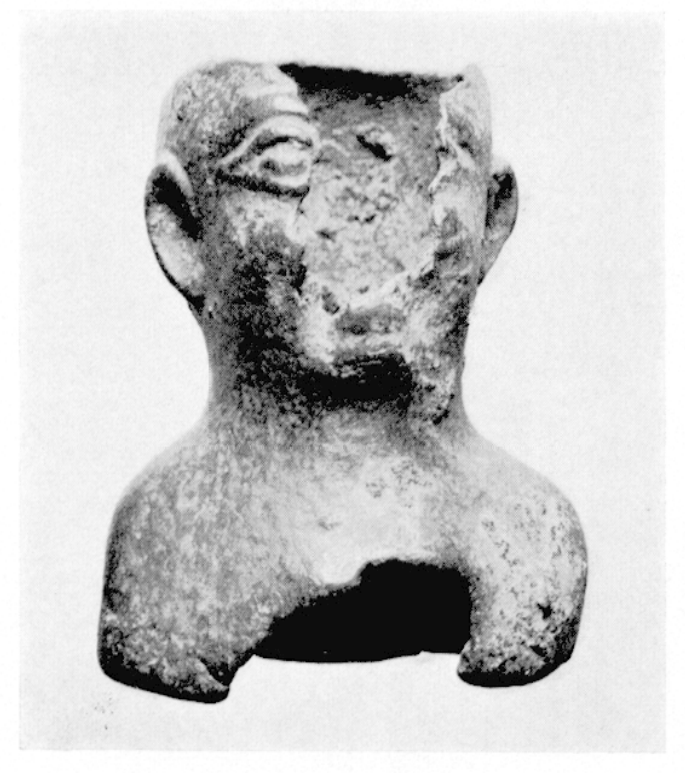

16 Vase plastique. Jyon, laalais des Bealux-.1rts.

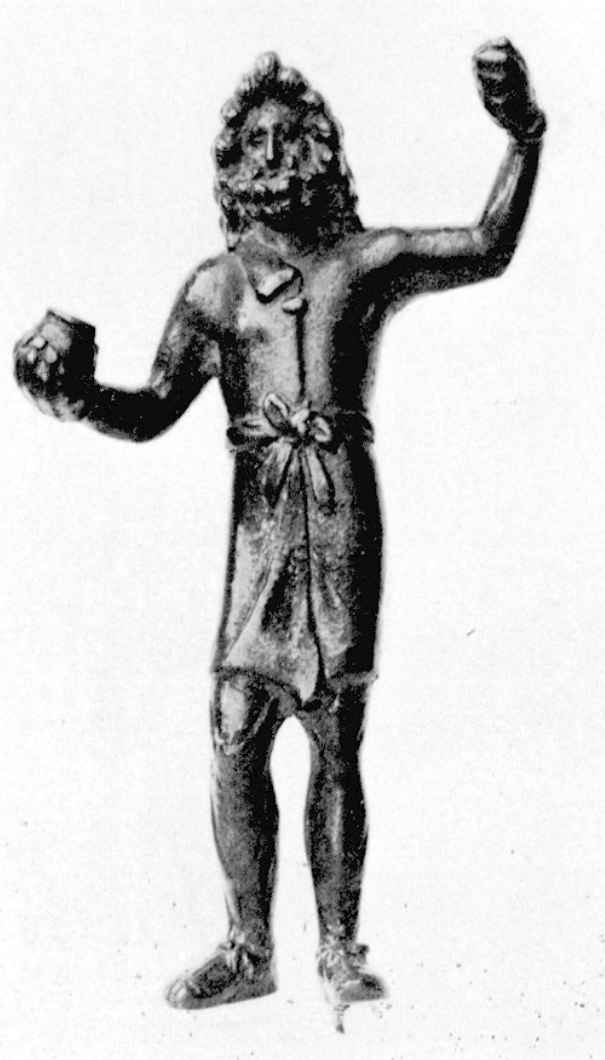

17 Dispmaler. Ixom, llusic de liourvieres. 


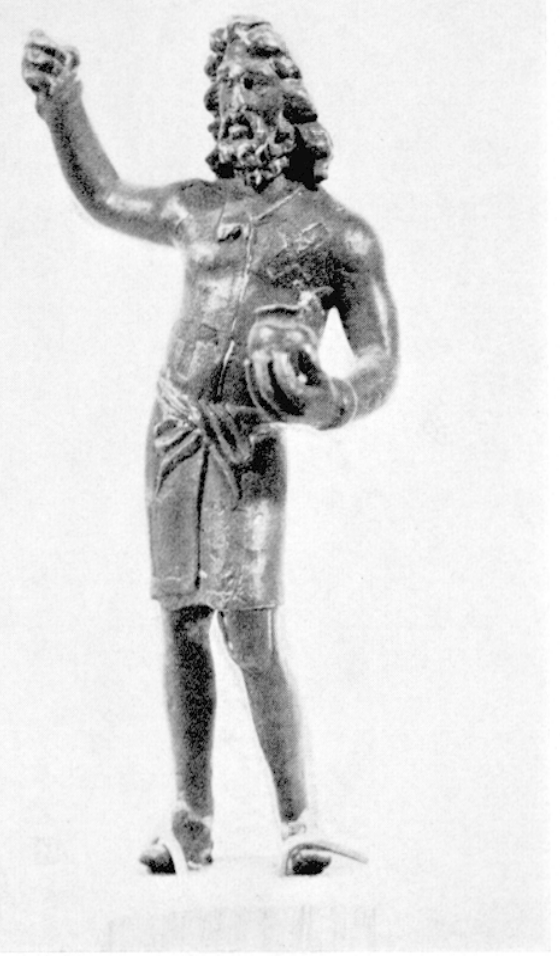

18 Iispaler. Lyon, Musée de Fourvières.

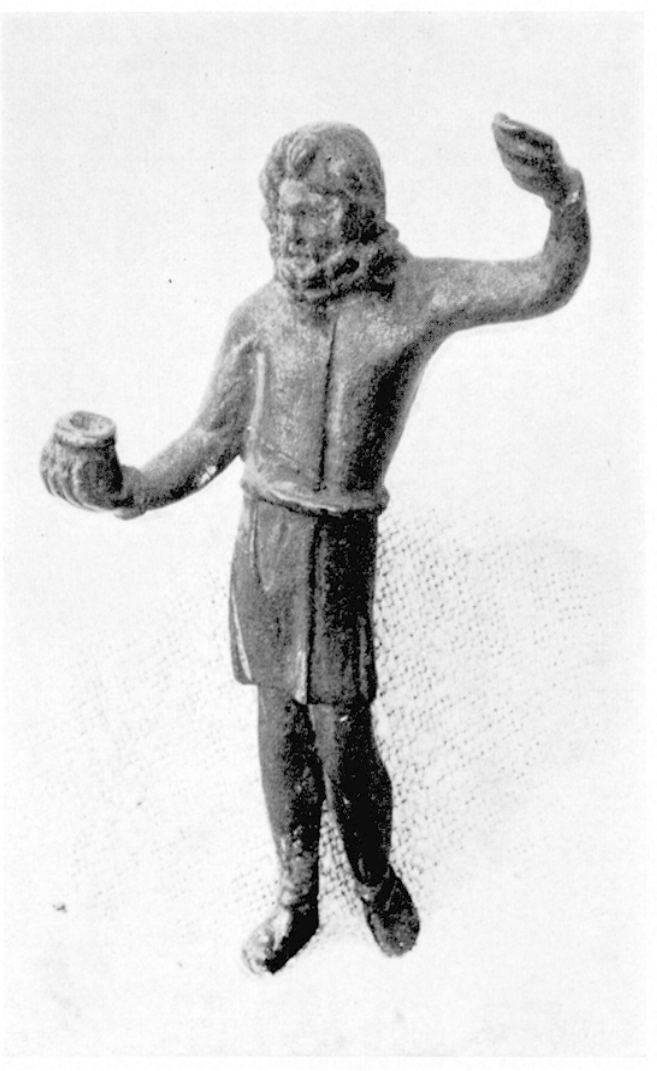

19 Dispaler. lyon, Musie de Fourvieres.

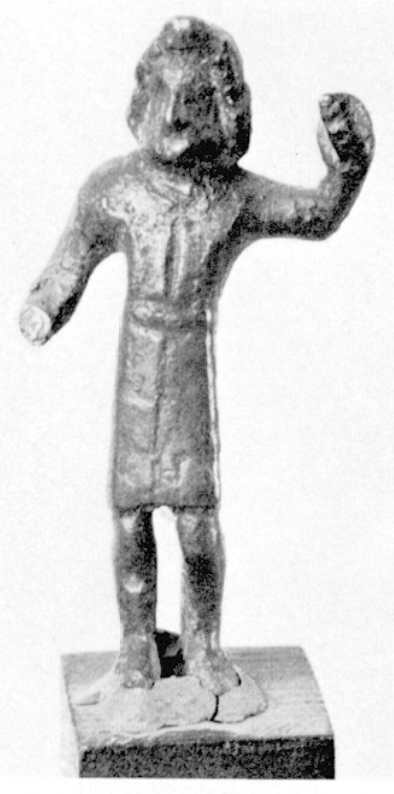

?II Dispaler. Lyon, Musée de Fourvières.

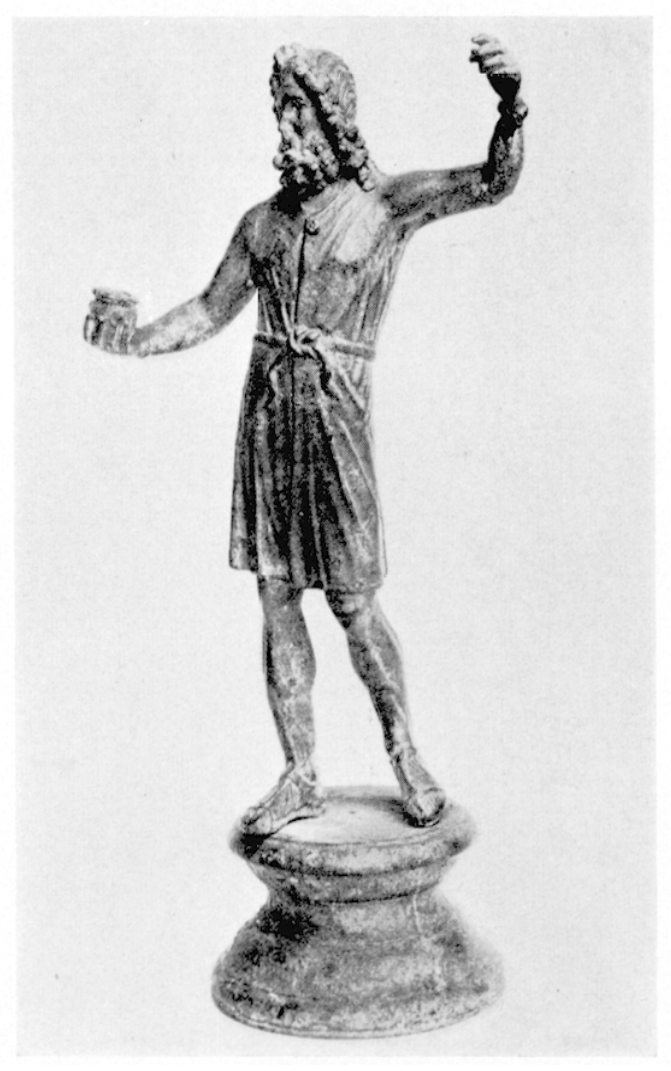

:I Dispater. Lyon, Musée de Fourvieres. 


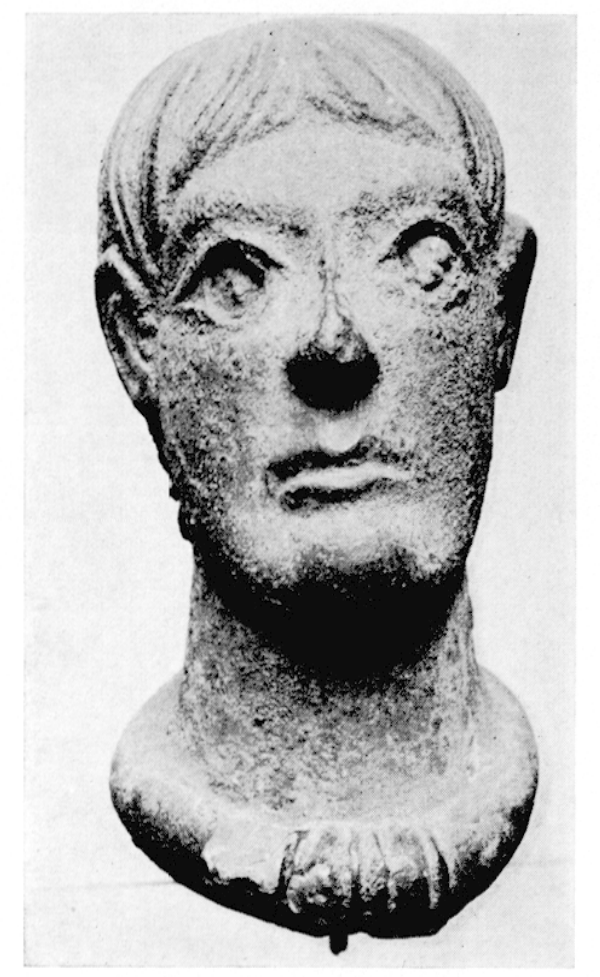

2: Tete de Gaulois. Lyon, Musée de Fourvières.

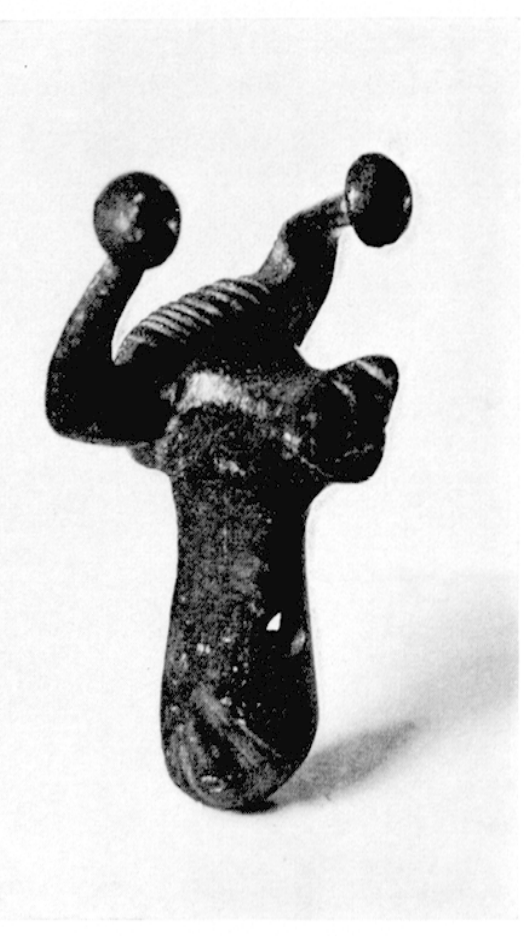

23 Tête de taureau à cornes bouletíes. Musie de Vimne. 